\title{
Obstáculos epistemológicos sobre los conceptos de límite y continuidad en cursos de cálculo diferencial en programas de ingeniería
}

\begin{abstract}
César Augusto Hernandez-Suárez ${ }^{\text {a*}}$, Raúl Prada-Núñez ${ }^{\mathrm{b}}$, Pastor Ramírez-Leal $^{\mathrm{c}}$
${ }^{a}$ Magister en Enseñanza de las Ciencias Básicas. Docente de la Facultad de Educación, Artes y Humanidades - Universidad Francisco de Paula Santander., (DD ' ${ }^{b}$ Magíster en educación matemática. Docente Tiempo Completo, Universidad Francisco de Paula Santander, (DD ${ }^{\mathrm{c}}$ Magíster en educación matemática, Docente Tiempo Completo UFPS. (ID

Forma de citar: Hernández, C. A., Prada, R. \& Ramírez, P. (2017). Obstáculos epistemológicos sobre los conceptos de límite y continuidad en cursos de cálculo diferencial en programas de ingeniería.
\end{abstract}

Perspectivas, 2(2). 73-83.

Recibido: marzo 08 de 2017

Aceptado: junio 21 de 2017

\section{Palabras clave}

Obstáculos epistemológicos, límite, continuidad.

\section{Keywords}

Epistemological obstacles, limit, continuity
Resumen: El objetivo de este trabajo fue identificar los obstáculos epistemológicos sobre los conceptos de límite y continuidad que presentan los estudiantes de programas de la Facultad de Ingeniería al inicio del proceso de formación académica en una universidad pública. Se diseñó un instrumento que incorpora una serie de actividades que utilizan diversos registros de representación semiótica tendientes a determinar el nivel de apropiación que poseen los estudiantes alrededor de los conceptos de límite y continuidad. De los hallazgos se resalta que los estudiantes asumen como iguales los conceptos de límite y continuidad.

\section{Epistemological obstacles on the concepts of limit and continuity in courses of differential calculus in engineering programs}

\begin{abstract}
Many investigations have been carried out with the objective of identifying the difficulties that students have in the learning process of the different mathematical concepts. Some studies have highlighted that the process of teaching mathematical knowledge by teachers in secondary and secondary education in Colombia, has been limited to a
\end{abstract} *Autor para correspondencia
cesaraugusto@ufps.edu.co 
minimalist expression of algebraic processes that in no way contribute to the understanding and appropriation of these concepts of origin abstract. Students who enter the various engineering programs in the university must immediately face a Differential Calculus course, which will demand from the student a whole series of competences around the numerical, variational and spatial thoughts. It is in this scenario where we seek to identify the epistemological obstacles presented by the students of the Faculty of Engineering programs at the beginning of the academic training process at the UFPS. An instrument was designed that incorporates a series of activities that use diverse registers of semiotic representation tending to determine the level of appropriation that the students have around the concepts of limit and continuity. From the findings it is highlighted that students assume the concepts of limit and continuity as equals.

\section{Palavras chave}

Obstáculos epistemológicos, limite, continuidade

\section{Obstáculos epistemológicos sobre os conceitos de limite e continuidade em cursos de cálculo diferencial em programas de engenharia}

Resumo: Muitas investigações foram realizadas com o objetivo de identificar as dificuldades que os alunos têm no processo de aprendizagem dos diferentes conceitos matemáticos. Alguns estudos enfatizaram que o processo de ensino de conhecimento matemático por professores em educação secundária e secundária na Colômbia tem sido limitado a uma expressão minimalista de processos algébricos que de forma alguma contribuem para a compreensão e apropriação desses conceitos de origem resumo Os estudantes que entram nos vários programas de engenharia na universidade devem enfrentar imediatamente um curso de Cálculo Diferencial, que exigirá do aluno toda uma série de competências em torno dos pensamentos numéricos, variacionais e espaciais. É neste cenário que buscamos identificar os obstáculos epistemológicos apresentados pelos alunos dos programas de Faculdade de Engenharia no início do processo de treinamento acadêmico na UFPS. Foi desenhado um instrumento que incorpora uma série de atividades que utilizam diversos registros de representação semiótica que tendem a determinar o nível de apropriação que os alunos têm em torno dos conceitos de limite e continuidade. A partir dos achados, destaca-se que os alunos assumem os conceitos de limite e continuidade como iguais. 


\section{Introducción}

Todo estudiante que ingresa al sistema de Educación Superior debe iniciar su formación académica superando, como mínimo un curso de Matemáticas en programas de formación sociohumanista, y dos o más cursos en programas de Ingeniería; cursos en los que se abordan diversos conceptos ya tratados en su formación en los niveles de básica y media. Pero el haberlos abordado anteriormente, pareciera que en ningún momento les garantiza la obtención de resultados favorables en la universidad; posiblemente debido a que la enseñanza se ha limitado a la realización de procesos mecánicos asociados con el desarrollo de competencias del pensamiento variacional.

Ante esta forma de enseñar Hitt (2003) afirma: "es difícil concebir que un alumno pueda entender el Cálculo sin haber desarrollado, por ejemplo, habilidades visuales ligadas a la construcción de conceptos". Con lo citado por Hitt (2003), se pone en evidencia que el uso recurrente de un único registro de representación en el proceso de enseñanza se convierte en un obstáculo en busca de la comprensión de los conceptos matemáticos, por tanto, se esperaría que un adecuado proceso de enseñanza en el aula debe propender por el uso y articulación coherente (libre de errores) de los diversos registros de representación semiótica.

Lo anterior, coincide con el interés que han mostrado los investigadores en el campo de la Educación Matemática con respecto al escaso dominio que poseen los estudiantes alrededor de los conceptos matemáticos al finalizar su proceso de formación en Educación Media; evidencia de esto son los resultados obtenidos por Colombia en pruebas internacionales como por ejemplo el Trend in International Mathematics and Science Study - TIMSS y Programme for International Student Assessment - PISA, donde el promedio de los estudiantes del país ha estado dominado por la media general de la prueba.

En éste sentido, toda investigación que se oriente a la identificación de dificultades de aprendizaje para mejorar la calidad de la educación siempre será de interés prioritario, por tanto todas las acciones orientadas a determinar dificultades de aprendizaje en los estudiantes, a identificar las buenas o no tan buenas prácticas docentes, al análisis del proceso evaluativo, a la caracterización de los recursos didácticos utilizados en el aula por parte de los docentes, entre otros aspectos; todos aportan información valiosa destinada a la transformación de las prácticas docentes y al mejoramiento del proceso enseñanza-aprendizaje.

Una pregunta por responder es ¿qué factores intervienen en los procesos de enseñanza que mejoran el aprendizaje de los estudiantes? Para iniciar el abordaje de este tema se cita a Santos (1994) quien señala que: "el tipo de actividades que se fomenten en el salón de clase necesariamente influyen en el tipo de aprovechamiento que los estudiantes exhiban en sus procesos de entendimiento o resolución de problemas".

En muchas ocasiones como lo menciona Hitt (2003), el docente presenta en el aula ejemplos asociados a situaciones de la vida real para introducir los conceptos matemáticos, pero si dichos ejemplos no han sido bien diseñados, tal es el caso de la siguiente redacción: la edad de un padre es equivalente al doble de la edad de su hijo aumentado en diez. El enunciado anterior propone una incoherencia lógica que no se ajusta a la realidad debido a que si el estudiante razona: cuando el hijo tiene edad de cero años su papá tendría entonces 10 años, luego este tipo de ejemplos que puede surgir de la necesidad del docente de presentar una situación en contexto en el aula (pero sin haberla preparado) seguramente desencadenaran en la apropiación limitada y posiblemente errónea en el estudiante.

Por ello se recomienda la utilización de ejercicios muy bien pensados que no propicien en inconsistencias para alcanzar el objetivo propuesto que es el de llevar a los estudiantes a un nivel más profundo de entendimiento del conocimiento dónde evidencien el campo de aplicación de los conceptos en situaciones cotidianas. Otro problema 
que se desprende de la incorporación de este tipo de situaciones no preparadas es generar en el estudiante la insensibilidad a incoherencias y contradicciones lógicas al obtener un resultado.

Con lo afirmado anteriormente se desea hacer énfasis en el hecho de que la presencia de dificultades en el manejo de los conceptos matemáticos no recae sólo en el estudiante y en sus capacidades, sino que el aprendizaje del estudiante termina siendo el producto de las dificultades conceptuales o las mismas concepciones presentes en los docentes que han orientado su proceso educativo.

Laintención de estainvestigaciónnoera analizar el problema del aprendizaje de las Matemáticas desde un enfoque global, sino profundizar sobre los conceptos de limite y continuidad, la cual complementa las investigaciones de Prada, Hernández, \& Jaimes (2017a, 2017b); Prada, Hernández \& Ramírez (2016); Hernández, Jaimes \& Chaves (2016) y Jaimes,Chaves\&Hernández (2015).

Otras Muchas investigaciones se han desarrollado alrededor de los conceptos de función, límite, continuidad y derivación. Por ejemplo, en los trabajos de Dreyfus y Eisenberg (1991), Dubinsky y Harel (1992), Hitt (2003),y Vallejo \& Pluvinage (s.f.) que ponen de manifiesto toda una diversidad de dificultades en el aprendizaje que distan de ser solucionadas cuando comienza la enseñanza del Cálculo.

\section{Materiales y métodos}

Este trabajo se ubica en el nivel de investigación descriptiva en la medición de variables independientes ya que se buscaba caracterizar las dificultades observadas en una población de individuos, resaltando en ellos aspectos como porcentajes de aciertos, desaciertos, actividades realizadas, herramientas utilizadas, entre otros. Se adopta el diseño de campo ya que la información será recolectada directamente de la fuente primaria como lo son los estudiantes.

\section{Población y Muestra}

La definición de la población se parte del marco referencial que está integrada por la totalidad de estudiantes matriculados en el curso de Cálculo Diferencial para el I semestre de 2017 de la Facultad de Ingenierías en la Universidad Francisco de Paula Santander sede Cúcuta, Colombia. No se consideró la aplicación de un proceso de muestreo puesto que se deseaba explorar todas las opciones que ofrece la Facultad de Ingenierías, alcanzando un total de 376 estudiantes.

\subsection{Elaboración y elaboración de instrumentos}

En cuanto al instrumento utilizado se recurrió a dos fuentes de información: a) fuentes secundarias a través de las consultas realizadas en la búsqueda de antecedentes así como en la revisión de libros de Cálculo y documentos de historia de las Matemáticas con el fin de evidenciar una lista de posibles dificultades de aprendizaje que se comportan como concepciones; b) fuentes primaria que consistió en la conformación de un panel de docentes que han orientado el curso de Cálculo Diferencial durante al menos dos años. A ellos se les consultó sobre las dificultades que presentaban los estudiantes al momento de evaluar los conceptos básicos del Cálculo Diferencial.

Una vez se tenía la información de las fuentes mencionadas se procedió a construir el instrumento. Se recurrió a la utilización de diversos registros de representación semiótica y estaba orientado a diversas características propias de cada concepto. Finalmente, su aplicación se realizó garantizando que al momento de llevarla a cabo ya los conceptos evaluados se hubiesen desarrollado al interior de sus cursos.

\section{Resultados y discusión}

Para la presentación de la información derivada de la aplicación de la prueba se utilizaron representaciones gráficas porcentuales 
acompañadas de una descripción detallada de los hallazgos en cada uno de los ítems de cada prueba.

Ítem 1. La temperatura en grados centígrados de un objeto en función del tiempot, medido en horas, está dado por la siguiente función: $\mathrm{T}(\mathrm{t})=\left(20 \mathrm{t}^{2}+10 \mathrm{t}+25\right) /\left(\mathrm{t}^{2}+6 \mathrm{t}+5\right)$. ¿Qué temperatura tendrá el objeto con el paso indefinido del tiempo?

Gráfico 1

Agrupación de estudiantes según su proceso de solución del ítem 1

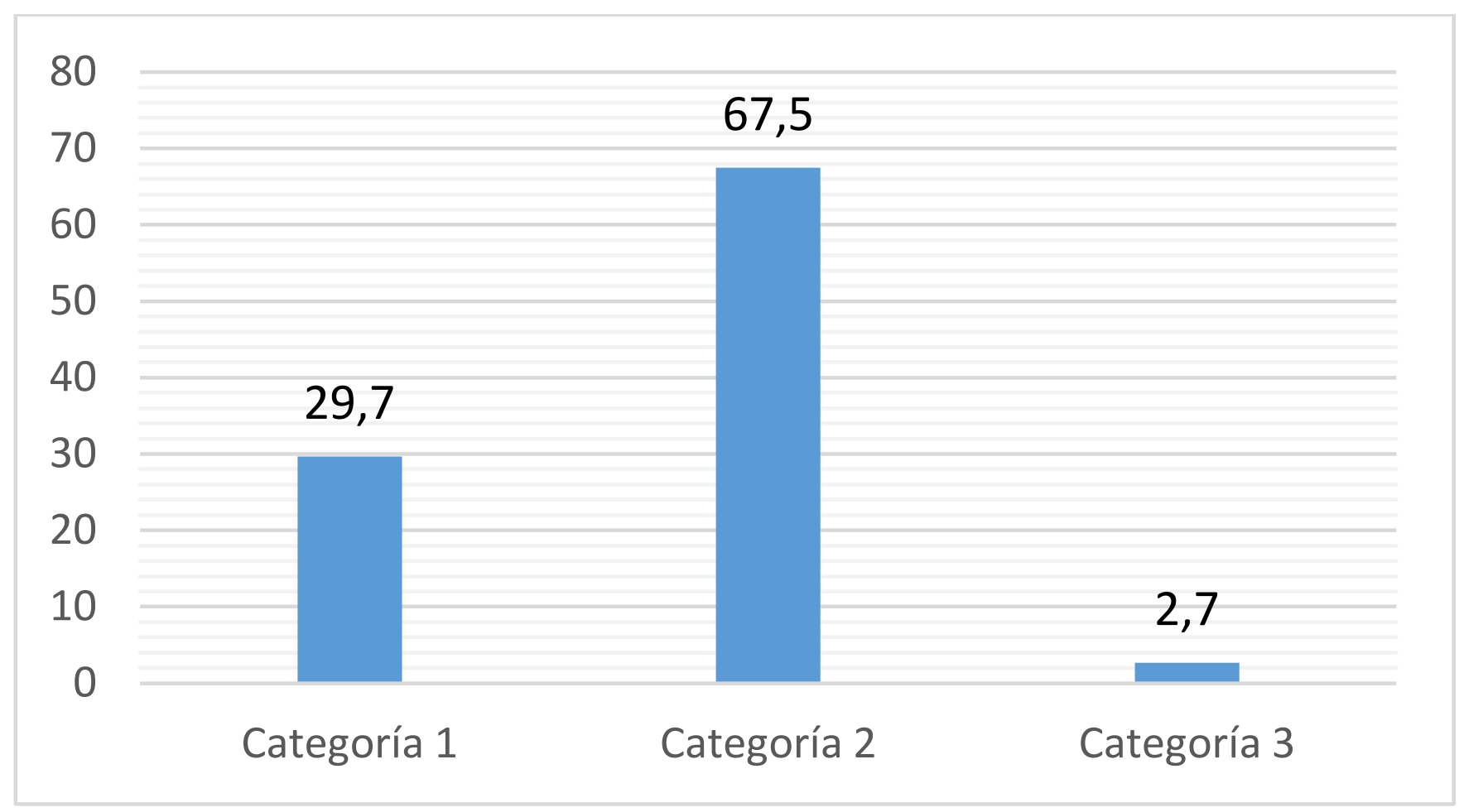

Fuente: Autor, Proceso de investigación.

El enunciado propone una situación en contexto con el fin de evaluar el límite en el infinito proporcionando la expresión algebraica asociada a la misma dónde la variable independiente corresponde al tiempo y la clave del enunciado se centraba en la frase "paso indefinido del tiempo". De los resultados se destaca que ningún estudiante resolvió la situación de forma completa y correcta, por ejemplo: los estudiantes agrupados en la Categoría 1, a partir de la expresión algebraica intentaron determinar el límite a través del registro tabular, pero evaluaron los cinco primeros números naturales, luego no les permitió llegar a una conclusión; en la Categoría 2 se ubica el predominio del grupo, todos intentaron resolver el límite de forma algebraica, pretendiendo factorizar cada término de la expresión (el denominador lo factorizan bien pero fallan con la expresión del numerador) con el fin de eliminar factores comunes, pero no superan la fase algebraica del ejercicio; finalmente en la Categoría 3 se ubican el grupo de estudiantes que al leer el enunciado interpretan adecuadamente la clave del enunciado, realizan el procedimiento algebraico de límites en el infinito, pero al llegar al valor de 20 consideran que esa es la respuesta, sin generar una conclusión en dónde se contextualice el valor obtenido. 
Ítem 2. Calcule el siguiente límite de forma tabular: $\lim _{x \rightarrow 3}\left(\frac{2}{x}+1\right)$

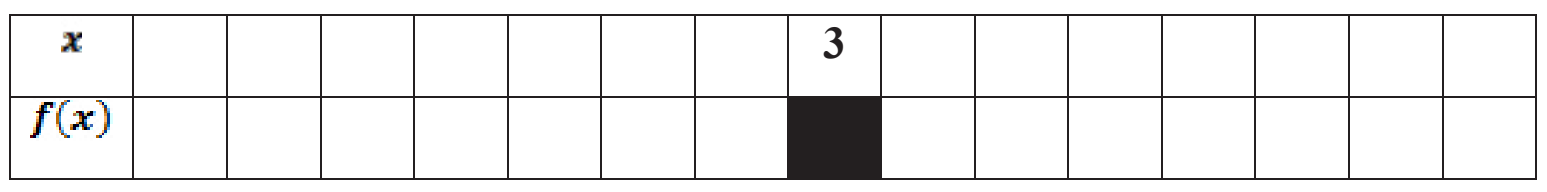

Gráfico 2

Agrupación de estudiantes según su proceso de solución del ítem 2

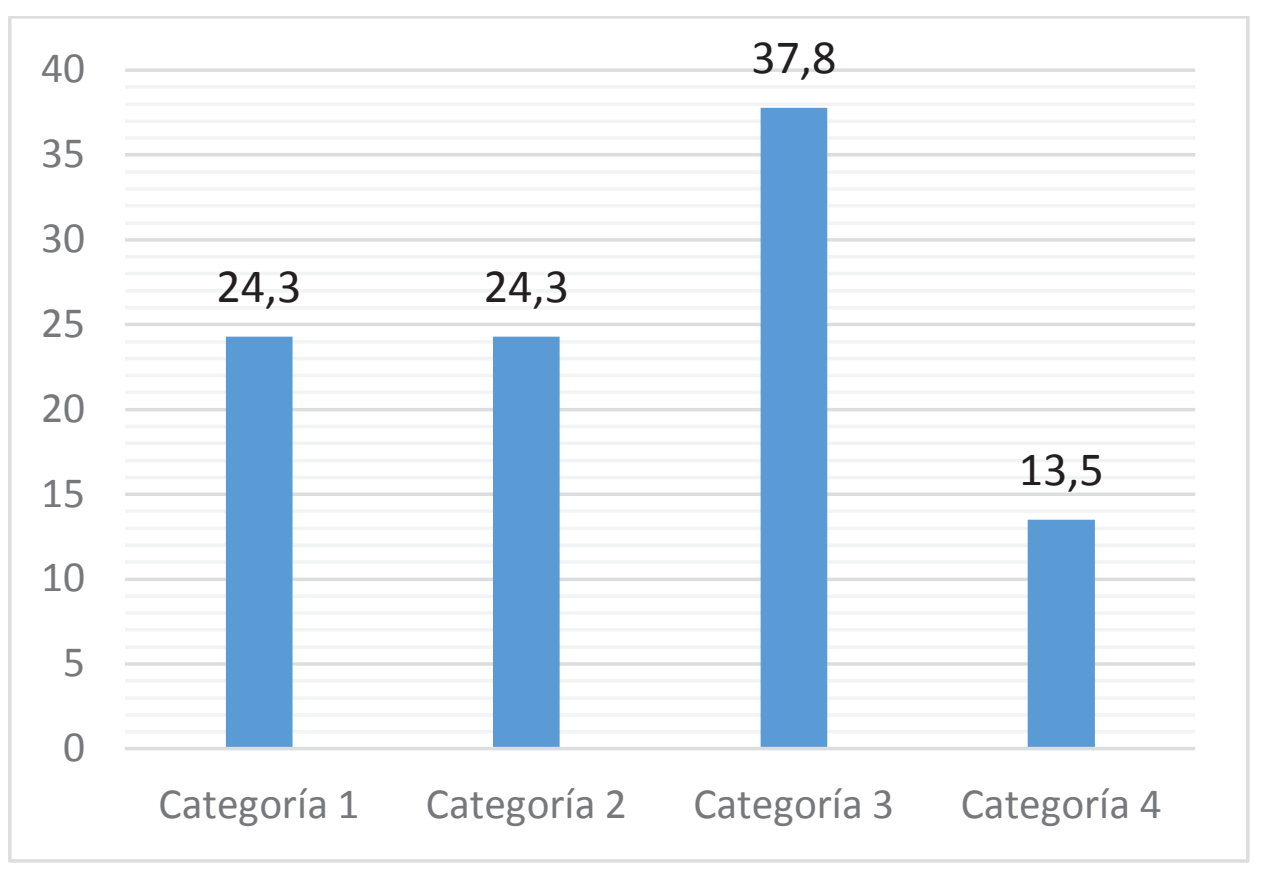

Fuente: Autor, Proceso de investigación.

El enunciado propone determinar el límite de una función utilizando el registro tabular, de los procesos realizados se destaca: en la Categoría 1 se ubican aquellos estudiantes que construyen de forma inadecuada la tabla de valores ya que asignan sólo valores enteros alrededor del 3 a la variable X desconociendo el concepto de límites laterales y el uso de valores infinitesimales; en la Categoría 2 se encuentran los estudiantes que al evaluar el límite en la tabla asignan adecuadamente los valores de $\mathrm{X}$ acercándose tanto por izquierda como por derecha a partir de una distancia máxima de una unidad, finalizando con la conclusión del límite a partir de verificar la igualdad de los límites laterales; en la
Categoría 3 están los estudiantes que desconocieron la indicación de calcular el límite de forma tabular, a pesar de que se les proporciona la tabla para su diligenciamiento, resolviendo el límite de forma algebraica realizando el proceso de sustitución de la variable $\mathrm{X}$ por el valor de tendencia; finalmente en la Categoría 4 están todos los estudiantes que construyeron adecuadamente la tabla para calcular el límite, evaluaron correctamente los valores asignados considerando la condición de máximo acercamiento al valor analizado, pero no concluyen el valor del límite, es decir, realizan sólo el proceso aritmético con los valores dados. 
Ítem 3. Determine la existencia del límite: $\lim _{x \rightarrow-2} \frac{x^{2}-3 x-10}{x^{2}-4}$

Gráfico 3

Agrupación de estudiantes según su proceso de solución del ítem 3

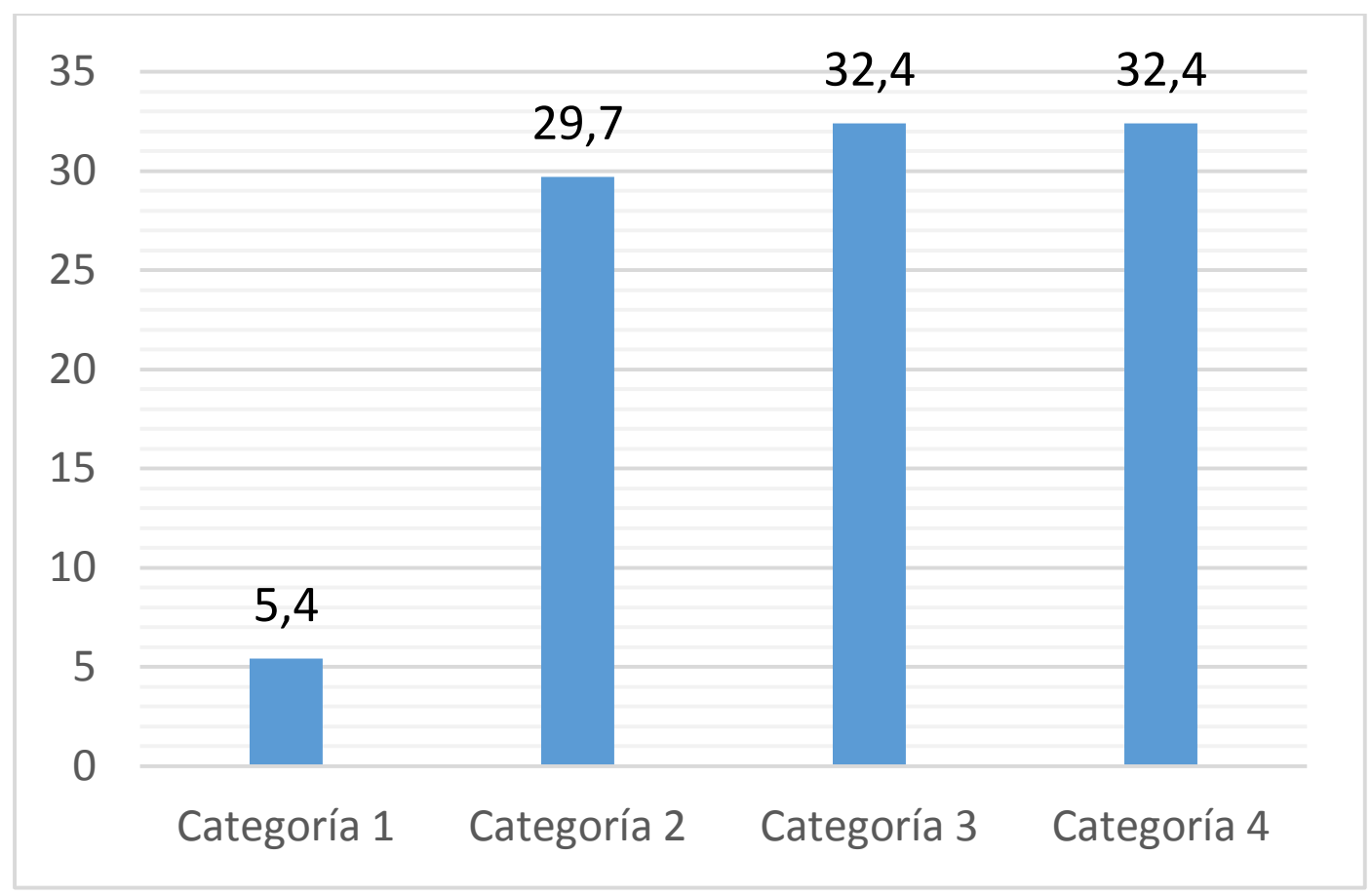

Fuente: Autor, Proceso de investigación.

El enunciado propone determinar el límite de una función a partir del registro algebraico, pero sin dar instrucciones del método a utilizar. De los resultados se generan cuatro categorías que se describen a continuación: en la Categoría 1 están los estudiantes que resolvieron el límite utilizando los registros tabular y algebraico, para concluir sobre la existencia del mismo a partir de la igualdad del resultado obtenido en ambos métodos; en la Categoría 2 se ubicaron los estudiantes que intentaron resolver primero el límite de forma tabular pero como seleccionan valores inadecuados no pueden llegar a ninguna conclusión, entonces luego proceden a resolverlo de forma algebraica dónde presentan dificultades en los procesos de descomposición factorial, para finalmente afirmar que el límite no existe; en la Categoría 3 están agrupados aquellos estudiantes que inicialmente buscan determinar el límite por medio del registro tabular pero fallan debido al uso inadecuado de valores, pero posteriormente resuelven de forma correcta el límite utilizando procesos algebraicos; por último, en la Categoría 4 se encuentran los estudiantes que resolvieron de forma adecuada el límite utilizando el registro tabular, pero al intentar determinar la solución de forma algebraica, cometen errores en los procesos de descomposición factorial después de verificar la condición de indeterminación de $0 / 0$ tras la evaluación del valor de tendencia. 
Ítem 4. Utilizando la información que le suministra la siguiente gráfica, determine el $\lim _{x \rightarrow 3} f(x)$

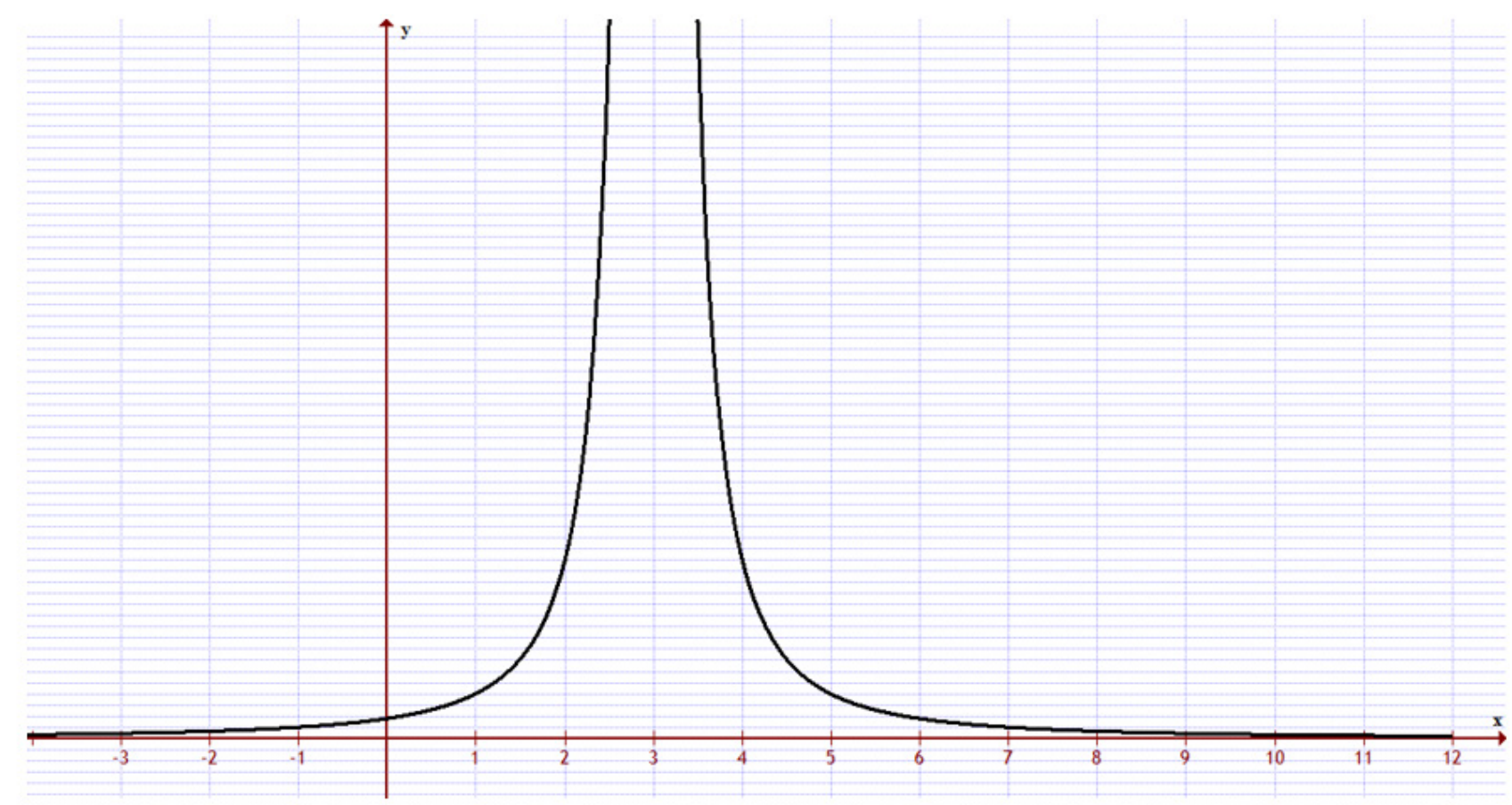

Gráfico 4

Agrupación de estudiantes según su proceso de solución del ítem 4

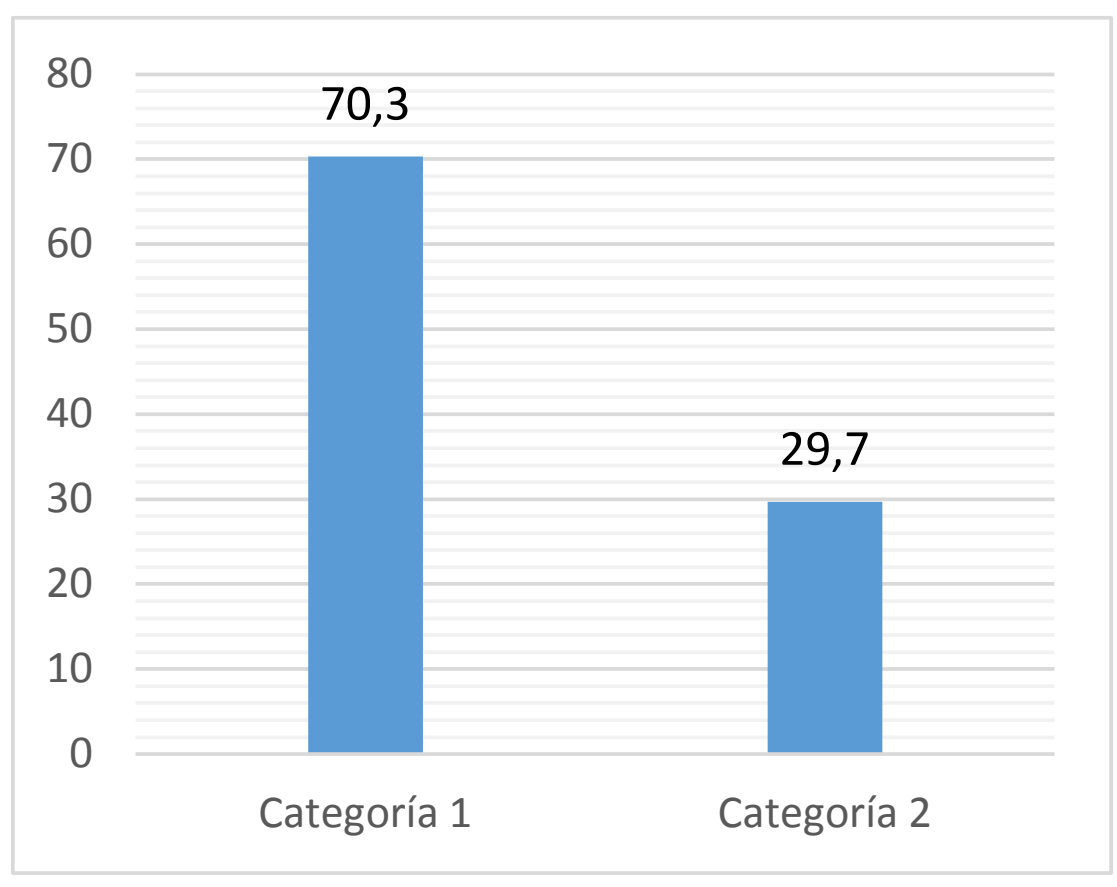

Fuente: Autor, Proceso de investigación. 
El enunciado propone determinar el límite de una función a partir del registro gráfico sin proporcionar expresión algebraica alguna. En la Categoría 1 se ubican la mayoría de los estudiantes evaluados, quienes afirman que nunca les habían solicitado determinar la existencia de un límite a partir de una gráfica y sin proporcionarles ninguna ecuación, luego por ello afirman que no saben cómo resolver éste tipo de situación propuesta; en la Categoría 2están aquellos estudiantes que a pesar de afirmar nunca haber trabajado con situaciones de este tipo, tímidamente afirman que el límite de la función en el valor indicado es de infinito. Situación que entra en conflicto con la condición de unicidad del límite.

Ítem 5. Evalúe la continuidad de la siguiente función cuando $\quad t=2: \quad g(t)=\frac{t^{3}-8}{t-2}$

Gráfico 5

Agrupación de estudiantes según su proceso de solución del ítem 5

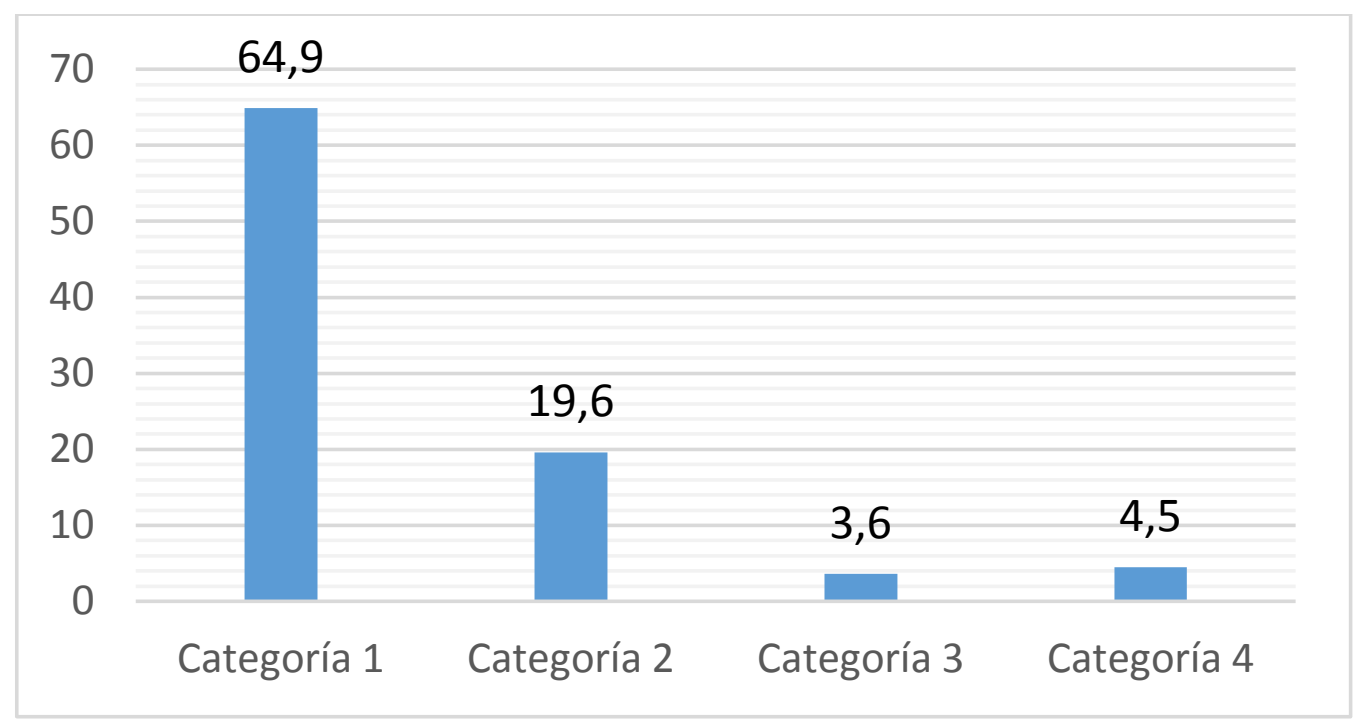

Fuente: Autor, Proceso de investigación.

El enunciado propone determinar la Continuidad de una función racional en un punto dado. En la Categoría 1 se agrupan los estudiantes que evalúan el valor dado en la función, al obtener la indeterminación $0 / 0$ no avanzan más; en la Categoría 2 están los estudiantes que afirmaron desconocer totalmente el tema de Continuidad ya que nunca les fue mencionado en las clases; la Categoría 3 la integran los estudiantes que después de evaluar el valor en la función afirman que se llega a una indeterminación que es propiciada por la existencia de un factor común entre el numerador y el denominador, por ello factoriza cada termino, simplifican y con la expresión reducida, calculan el límite en $\mathrm{t}=2$. Finalmente, afirmar que la función presenta una discontinuidad evitable o removible pero no propone una expresión algebraica que garantice la condición de continuidad en el valor indicado; finalmente en la Categoría 4están los estudiantes que factorizan la expresión dada, luego determinan el límite de la función reducida en $\mathrm{t}=2$, obtienen un valor y dan por terminada la solución, sin hacer comentario alguno sobre el proceso de continuidad. 


\section{Conclusiones}

El tema de límite de una función es abordado por el docente casi siempre de forma algebraica centrada en la simple sustitución del valor de tendencia en la expresión, concepción que resulta válida hasta el momento en que el estudiante se tiene que enfrentar a expresiones con límites factorizables, luego allí empiezan a surgir inconvenientes debido a la baja competencia que poseen los estudiantes en los procesos de descomposición factorial. Esta dificultad es evitable si al momento de explicar el tema el docente incorporara diversos registros de representación semiótica de forma que resulten complementarios unos con otros, pero que parta siempre del concepto de aproximación por los dos lados, es decir, verificar la condición de unicidad del límite.

En cuanto al tema de continuidad de funciones se evidencia desconocimiento en los estudiantes en el proceso a seguir en cuanto la evaluación de esta, se evidencia dificultad para determinar el tipo de discontinuidad que presenta una función, que sumado al desconocimiento de las funciones definidas por partes resulta inoperable la determinación de una adecuada solución cuando la discontinuidad es evitable.

Finalmente, los estudiantes confunden los conceptos de Límite y Continuidad asumiendo que los dos representan la misma idea, lo que se convierte en un obstáculo conceptual. En sus procesos argumentativos presentan dificultad para evaluar la existencia del límite en un punto, lo que les impide así mismo determinar el tipo de discontinuidad que se pueda presentar. Los hallazgos encontrados en esta investigación, sugieren que los docentes deben implementar secuencias didácticas que vayan aumentando gradualmente la complejidad de las situaciones, al tiempo que utilizan diversos registros de representación.

\section{Referencias}

Dreyfus, T. y Eisenberg, T. (1991). On the reluctance to visualize in mathematics. EnW. Zimmermann y S. Cunningham (Eds.). Visualization in teaching and learning inmathematics (pp 25-38). MAA Notes No. 19

Dubinsky, E., y Harel, G. (1992). The Concept of function: aspects of epistemology and pedagogy. Washington, DC: Mathematical Association of America.

Hernández, C. A., Jaimes, L. A. \& Chaves, R. F. (2016). Modelos de aplicación de ecuaciones diferenciales de primer orden con Geogebra: actividades para resolver problemas de mezclas. Mundo Fesc. 11, 7 - 15.

Hitt, F. (2003). Dificultades en el aprendizaje del Cálculo. In XI Meeting of Middle-Higher Level Mathematics Teachers, Michoacan University San Nicolás de Hidalgo, Morelia (Mexico).

Jaimes, L. A. Chaves, R. F. \& Hernández, C. A. (2015). Planteamiento de una ecuación diferencial lineal de primer orden que modela un problema de mezclas: Una dificultad en la movilización entre registros de representación, lengua natural y algebraico. Elementos. 5, 23 31.

Prada, R. Hernandez, C. A. \& Jaimes, L. A. Contreras (2017a). Representación semiótica de la noción de función: concepciones de los estudiantes que transitan del Colegio a la Universidad. Panorama. 11(20), 34 - 44, doi: http://dx.doi. org/10.15765/pnrm.v11i20

Prada, R. Hernandez, C. A. \& Jaimes, L. A. Contreras (2017b). Representaciones semióticas alrededor del concepto de función en estudiantes de ingeniería. Revista Góndola, Enseñanza Y Aprendizaje De Las Ciencias. 12(2), 32 - 49. doi: 10.14483/udistrital.jour.gdla.2017.v12n2. a2

Prada, R. Hernandez, C. A. \& Ramírez, P. (2016). Comprensión de la noción de función y la articulación de los registros semióticos que la representan entre estudiantes que ingresan a un programa de Ingeniería. Revista Científica. 2(25), 45 - 72. doi: http://dx.doi.org/10.14483// udistrital.jour.RC.2016.25.a3

Santos, L. (1994). La resolución de problemas en 
el aprendizaje de las matemáticas. Cuadernos de investigación. Departamento de Matemática Educativa. CINVESTAV-IPN, México.

Vallejo, C., \& Pluvinage, F. Investigaciones sobre la enseñanza del Cálculo. 